\title{
Quelles identités culturelles pour les jeunes de l'Océan Indien ?
}

Pascal Duret, Muriel Augustini, Georges Dalleau, Pierre Leroyer, Maurice Zattara

\section{Citer ce document / Cite this document :}

Duret Pascal, Augustini Muriel, Dalleau Georges, Leroyer Pierre, Zattara Maurice. Quelles identités culturelles pour les jeunes de l'Océan Indien ?. In: Agora débats/jeunesses, 20, 2000. Résistances à l'uniformisation culturelle. pp. 13-22; doi : https://doi.org/10.3406/agora.2000.1747

https://www.persee.fr/doc/agora_1268-5666_2000_num_20_1_1747

Fichier pdf généré le 05/04/2018 


\title{
Résumé
}

Cet article souhaite prendre de la distance avec deux types de regards ; d'une part, celui ne percevant que les similitudes chez les jeunes des îles du sud-ouest de l'Océan Indien et, d'autre part, celui suggérant au contraire qu'ils n'ont rien à partager. Ni naïvement apologétique, ni désespérément sceptique, il s'attache aux avancées d'un bassin culturel en train de se faire. II analyse la place réservée aux consommations de loisirs pour ces jeunes. Enfin, il analyse le rôle tenu par les manifestations sportives dans le bassin culturel, qui à la fois joue dans la mise en place de relations fraternelles, mais rappelle aussi à chacun des pays son niveau de développement.

\begin{abstract}
On the Etang Salé beach (Reunion), on Flic en Flac beach (Mauritius), on Praslin beaches (Seychelles) or even on the large coastal stretches close to Tamatave (Madagascar), the same young people seem to be playing the sames games while listening to the same music. Everything contributes to this lack of differentiation, aren't these island considered as "Sister islands ? The aim of this paper is to invite the reader to take part in an unusual game of «spot the differences ", as an antidote against the standardized vision projected by cliches of deceptive exotism.
\end{abstract}

\section{Resumen}

En la playa de la Laguna Salada (Reunión), al igual que en la del «Flic en Flac» (Mauricio), como en las de Praslin (Seychelles), o también en las inmensas extensiones costeras cercanas a Tamatave (Madagascar), parece que veamos a los mismos jóvenes jugando a los mismos juegos y escuchando la misma música. Todo conspira a este efecto de falta de diferenciación, ¿ no se habla de «islas hermanas» ? El propósito de este artículo es, en primera instancia, invitar al lector a participar en un insólito «juego de los cinco errores», como antídoto a la visión uniformizante proyectada por los tópicos de un exotismo engañoso.

\section{Zusammenfassung}

Am Strand von Etang Salé (Réunion) wie am Strand von Flic en Flac (Mauritius) oder am Strand von Praslin (Seychellen) sowie an den riesengrossen Küstenstreifen in der Nähe von Tamatave (Madagascar) sind es anscheinend die gleichen Jugendlichen, die die gleichen Spiele haben und dabei die gleiche Musik hören. Alles trägt zu dieser Undifferenzierungswirkung bei, spricht man nicht übrigens von den «Schwesterinseln «? Ziel dieses Artikels ist zuerst den Leser an einem «Spiel der fünf Irrtümer «teilnehmen zu lassen und zwar als Gegenmittel zur vereinheitlichenden Vision, die von den Klischees einer teuchenden Exotik hervorgerufen wird. 


\section{Quelles identités culturelles pour les jeunes de l'Océan Indien?}

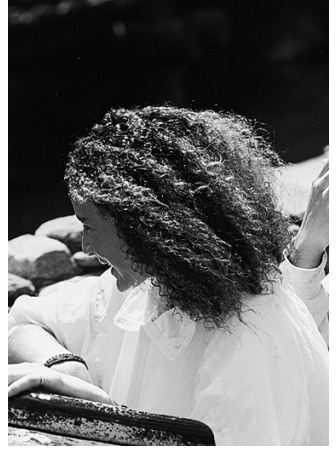

Pascal Duret,

Muriel Augustini,

Georges Dalleau,

Pierre Leroyer,

Maurice Zattara,

Enseignants/chercheurs au CURAPS,

Université de La Réunion.

117, rue du Général Ailleret

97430 Tampon - La Réunion

Pascal.Duret@univ-reunion.fr

Cet article souhaite prendre de la distance avec deux types de regards ; d'une part, celui ne percevant que les similitudes chez les jeunes des îles du sud-ouest de l'Océan Indien et, d'autre part, celui suggérant au contraire qu'ils n'ont rien à partager. Ni naïvement apologétique, ni désespérément sceptique, il s'attache aux avancées d'un bassin culturel en train de se faire. II analyse la place réservée aux consommations de loisirs pour ces jeunes. Enfin, il analyse le rôle tenu par les manifestations sportives dans le bassin culturel, qui à la fois joue dans la mise en place de relations fraternelles, mais rappelle aussi à chacun des pays son niveau de développement.

\author{
par Pascal DURET, Muriel AUGUSTINI, \\ Georges DALLEAU, Pierre LEROYER, \\ et Maurice ZATTARA.
}

Tout vacancier peut facilement se laisser abuser par une ressemblance de surface entre les différentes jeunesses qui peuplent les îles de l'Océan Indien. Sur la plage de l'Étang Salé (Réunion), comme sur celle de Flic en Flac (Maurice), comme sur celles de Praslin (Seychelles), ou encore comme sur les immenses étendues côtières proches de Tamatave (Madagascar), il semble que se soient les mêmes jeunes qui jouent aux mêmes jeux en écoutant la même musique. Tout conspire à cet effet d'indifférenciation, ne parle-t-on pas des “ îles sœurs ”. Le but de cet article est tout d'abord d'inviter le lecteur à participer à un insolite " jeu des cinq erreurs ", comme antidote à la vision uniformisante projetée par les clichés d'un exotisme trompeur. Dans un second temps, nous identifierons quelques obstacles rencontrés dans la construction de cette région du monde en une zone économique et en bassin culturel.

\section{Les jeux des cinq erreurs}

Première erreur. L'Océan Indien ne constitue pas un tout unifié, ainsi convient-il de distinguer les îles du Sud-ouest de l'Océan 
Indien (les Comores, Madagascar, Maurice, La Réunion) de celles plus aux nord (comme les Maldives) ou celles toutes proches de la côte africaine (comme Zanzibar). Même à l'intérieur du Bassin des Mascareignes, dont la francophonie (Chaudenson, 1992, Maestri, 1994) et (d'une manière plus relative) la créolophonie constituent d'importants facteurs d'unité (Chaudenson, 1995) on repère des différences sensibles entre les styles de vie des jeunes et dans leur sentiment d'appartenance à une même région du monde.

Revenons à notre vision des plages pour les analyser. Les jeunes mauriciens ne disposent pas, par exemple, des mêmes espaces publics que ceux de La Réunion. S'ils sont tassés à Flic en Flac c'est qu'il s'agit d'une des rares plages publiques de l'île, la plupart étant réservées aux clients des chaînes hôtelières. Plus les écarts sociaux se creusent moins il semble probable que des jeunes de différents milieux se fréquentent. Il est, par exemple, totalement invraisemblable que des Mauriciens créoles d'origines modestes fréquentent le très chic "Dodo club". Cette logique ségrégative très marquée dans les îles les plus pauvres (Comores, Madagascar) au point que la grande majorité des jeunes autochtones n’ont pas de " temps à perdre " à la plage ${ }^{1}$ est très largement atténuée au point de disparaître à La Réunion même si les plages des "roches noires" et de "Boucan canot" ne recrutent pas les mêmes assidus que celle de Manapagny ou de St-Pierre.

\footnotetext{
${ }^{1}$ Puisque dès le plus jeune âge il contribue à la cellule de production familiale. Le droit au loisir passe après la nécessité de la survie.

${ }^{2}$ Composées de plus d'une centaine d'îles les Seychelles sont peuplées de 75000 habitants concentrés quasi exclusivement sur trois d'entre elles Praslin, Mahé et la Digue.
}

Deuxième erreur. À y regarder de plus prêt, on aurait de fortes chances de s'apercevoir que les jeunes jouant sur la plage de Praslin ne sont pas originaires des Seychelles mais venus y passer des vacances. Les Seychelles ont en effet la particularité de recevoir annuellement plus de touristes qu'elles n'ont d'habitants $^{2}$. De fait, les jeunes des Mascareignes vivent dans des univers économiques très différents. La principale expérience de vie de ceux des Seychelles, mais plus encore des Malgaches et des Comoriens est la précarité. Vivre proche ou sous le seuil de pauvreté condamne à un quotidien où la mobilité inter-îles n'est pas monnaie courante. Un autre constat complémentaire s'impose lorsqu'on quitte Madagascar ou les Comores pour venir à La Réunion ce n'est pas pour une quinzaine touristique mais pour travailler et souvent à n'importe quel prix (ou plus rarement pour suivre des études).

Inversement, doté d'un bien meilleur niveau de vie, les jeunes réunionnais vont faire du tourisme (et du shopping) dans les îles voisines (Maurice surtout Madagascar parfois) au coût de la vie bien moindre que chez eux. Ces échanges inégalitaires ne contribuent pas à rapprocher ces différentes jeunesses.

Se développe à La Réunion même un état d'esprit “ anti-comorien ”. Bien évidemment, personne à La Réunion ne revendique la discrimination ethnique : au contraire, l'un des mythes fondateurs qui sert de ciment au lien social réunionnais est le refus du racisme qui se traduit en pratique par des scores jusque-là dérisoires du FN sur l'île (même si l'argument “ droit à la différence culturelle " et l'affirmation de la créolité s'emploient parfois 
de manière ambiguë). Cependant la peur du non emploi (taux de chômage de 34 \% sur l'île) ravive les logiques de préférence communautaire. À y regarder attentivement ce n'est pas véritablement sur le marché de l'emploi que les Comoriens sont perçus comme une menace par les jeunes réunionnais, mais bien plutôt en tant qu'agents de dérégulation du travail au noir ; ils sont, en effet, réputés “ casser les cours" en acceptant n'importe quel travail sans jamais discuter des conditions fixées par l'employeur.

\begin{tabular}{|l|l|l|l|l|l|l|}
\hline à La Réunion & 1992 & 1993 & 1994 & 1995 & 1996 & 1997 \\
\hline Demandes d'emplois & 80120 & 80200 & 85623 & 88837 & 96330 & 100482 \\
\hline Offres d'emplois & 14315 & 12529 & 20475 & 24503 & 34444 & 33224 \\
\hline
\end{tabular}

Source: Insee territoires 1997-1998.

Les moqueries stigmatisantes fournissent la preuve de cette animosité. Alors que l'humour inter-ethnique reste dans la grande majorité des situations assez "bon enfant" sans se priver pour autant d'épingler des stéréotypes de chacune des communautés, la communauté comorienne, elle, est raillée sans ménagement et il n'est sans doute pas excessif de parler de quasi-racisme à son égard. Les blagues dont font, par exemple, les frais les Zoreilles s'inscrivent dans une réciprocité et un toujours possible changement de rôles entre moqueurs et moqués. Le Créole se moque volontiers du Zoreille dépeint (hâtivement) comme un nanti profiteur mais à son tour celui-ci peut rire du Créole en le décrivant (tout aussi caricaturalement) comme un pur assisté. Les Comoriens, eux n'ont pas cette possibilité, ils sont toujours dans le camp des moqués.

Il convient également de retenir en matière de mobilité les statistiques les plus fréquemment mobilisées concernent soit les pratiques de loisirs, soit les statistiques d'arrivée aux frontières des travailleurs. La Réunion se situe encore largement en retrait en matière d'accueil touristique par rapport aux autres îles de la zone des Mascareignes ${ }^{3}$.

\begin{tabular}{|l|l|l|l|}
\hline & Île Maurice & Seychelles & La Réunion \\
\hline Part du tourisme dans le P.I.B. & $8,2 \%$ & $34,2 \%$ & $2,9 \%$ \\
\hline Coefficient de valeur ajoutée nationale & 0,90 & 0,67 & 0,43 \\
\hline
\end{tabular}

Source : Tourisme et développement, Observatoire du développement de La Réunion n 34, avril 1998

Par contre, les choix hypothétiques de vacances des jeunes réunionnais montrent l'ambiguïté de la référence à Paris. Nous avons administré un mini-questionnaire à 220 étudiants du campus de Saint-Denis. Une question leur demandait où ils aimeraient partir durant leurs vacances. Les réponses attestent de l'attachement à (ou de l'attrait exercé par) la métropole (et plus précisément par Paris). Mais une question ouverte destinée à éclairer ces choix montre paradoxale-

\footnotetext{
${ }^{3}$ Autre manière de lire les données qui aboutit à un autre constat : l'ensemble de l'économie réunionnaise s'est développé au même rythme que le tourisme, ce qui explique que son poids relatif n'ait pas progressé. II n'empêche que même comparativement à la moyenne nationale $(7 \%)$ le P.I.B. du tourisme réunionnais reste relativement faible.
} 


\section{On repère des différences sensibles entre les styles de vie des jeunes et dans leur sentiment d'appartenance à une même région du monde.}

tion est suffisamment intégrée dans les mœurs ${ }^{4}$ pour être chantée par les poètes locaux : ment que se rendre à Paris ne signifie pas pour eux vouloir visiter les hauts lieux de la culture française mais fréquenter ceux de la culture "Mc Monde" tels "Disneyland" ou “Planète Hollywood ”. Il s'agit à un deuxième niveau d'aller retrouver une partie de sa famille vivant en métropole, en aucun cas d'aller y chercher des racines identitaires nationales.

Choix de lieu des destinations de vacances des jeunes étudiants réunionnais : Les dix réponses les plus fréquentes exprimées en pourcentage

\begin{tabular}{|l|l|}
\hline Métropole & $27 \%$ \\
Maurice & $21 \%$ \\
Rester à La Réunion & $14 \%$ \\
Seychelles & $6 \%$ \\
Afrique du Sud & $5 \%$ \\
Madagascar & $4 \%$ \\
Amérique du Nord & $4 \%$ \\
Australie & $3 \%$ \\
Inde & $2 \%$ \\
Les Antilles & $2 \%$ \\
\hline
\end{tabular}

Troisième erreur. Tout observateur sur les plages de La Réunion peut constater que l'animation des heures chaudes (surf, beach volley...) laisse place en soirée à certaines périodes de l'année à un autre type d'effervescence, celle des feux de camp où les jeunes tout en écoutant ou en jouant de la musique fument souvent sans retenue le Zamal (le cannabis local), dont la consomma-
“ Oh Zamal, Oh Zamal tu fais mal à mon cœur ”. Sur les autres îles des Mascareignes consommer du cannabis, qu'il soit appelé Rongony par les Malgaches ou Banguey par les comoriens, passe souvent pour un forfait majeur et ceux qui enfreignent cet interdit risquent des peines sans aucune commune mesure avec celles encourues par les jeunes réunionnais. Il s'agit à Maurice d'un véritable crime passible d'emprisonnement à vie, les affiches en ville et à l'aéroport ne laissent aux éventuels contrevenants aucun doute sur la gravité de la sanction qui les attend. En outre la législation pénale ne peut être confondue à Madagascar avec l'application de la justice qui passe aussi par des pratiques populaires ou coutumières se substituant volontiers à l'État et à son droit constitutionnel perçu comme inefficace. Dans certaines parties de la "grande île" les jeunes délinquants et/ou toxicomanes s'exposent, s'ils sont pris sur le fait, à une lapidation pure et simple sans autre forme de procès (Ramilison, 1993). Du coup, les fêtes ne sont pas du tout marquées d'une île à l'autre par la même ambiance. Une jeune Mauricienne venue s'installer à La Réunion pour ses études en STAPS résume d'une expression ce décalage culturel “ La

\footnotetext{
${ }^{4}$ L'enquête INSERM 1997 sur l'usage des psychotropes dans les DOM situe le taux de contact avec les drogues douces à un jeune sur trois (usage beaucoup plus élevé qu'à la Martinique ou à la Guyane). Une enquête "Baromètre santé " menée actuellement par la DRASS devrait permettre d'affiner ces premiers résultats (cf. Infostat, Bulletin d'information de la DRASS $n^{\circ} 50$, juin 1999).
} 
Réunion quand on arrive de Maurice c'est la Jamaïque ". Rien de plus faux pourtant que de conclure à un laisser-aller des pouvoirs publics et des forces de l'ordre quand à l'application de la loi. Bien au contraire c'est parce que des campagnes de prévention efficaces et une surveillance intraitable (par hélicoptère notamment) ne laissent aucune chance à des plantations clandestines à grande échelle, que l'usage privé, presque " décoratif" du pied de Zamal au fond d'un jardin peut parfois persister. Ne pouvant aussi sûrement contrôler la situation sur le terrain de la toxicomanie, les autres pays de la région doivent agiter une menace répressive d'intensité supérieure.

Les modalités intergénérationnelles des fêtes elles aussi différent. À La Réunion, les fêtes organisées par les jeunes sont souvent des fêtes entre jeunes. À Maurice, les fêtes sont presque toujours une occasion d'entretenir les liens de la famille, les membres, cela va de soi, y sont présents. La famille est le lieu de la construction de soi et il n'y a pas à Maurice de "soi " sans " nous". L'identité individuelle de chaque jeune puise ses ressources au sein de ce "nous familial". Une autre étudiante mauricienne venue à $\mathrm{La}$ Réunion nous confiait : "Ce qui m'a le plus surpris lors de mes premières fêtes c'était l'alcool et le Zamal mais aussi le fait qu'il n'y avait jamais les parents, à Maurice si tu fais une fête tout le monde de ta famille participe, moi je danse avec mes grands-parents, les jeunes et les vieux sont ensemble, moi je préfère ". Les manières de vivre les rapports inter-générationnels au sein d'une même famille ne supposent donc pas à Maurice la séparation des activités, alors qu'elles l'exigent plus souvent à La Réunion. À Maurice, la famille ne s'impose pas, elle est là ; de même, on ne fait pas la fête avec sa famille pour s'assurer de son soutien logistique à l'organisation, ni pour suivre précisément une coutume, mais parce que l'on vit ensemble. À Madagascar s'ajoute à la proximité des ascendants le poids des ancêtres $\left(\right.$ razana $\left.^{5}\right)$. À La Réunion, les jeunes ont beau "vivre avec" et être " descendant de", la famille est plus traversée par des relations d'affinités électives, les “ anciens" ne jouent pas au sein de la famille le même rôle (Cheynel, 1985).

Quatrième erreur. Sur ces plages, les jeunes ne font pas tous ou n'écoutent pas tous les mêmes musiques. Ce sont, en effet, les musiques locales qui occupent une place déterminante. On repérera en particulier pour La Réunion :

- le Zouk et le Zouk love qui entretiennent une passerelle musicale entre La Réunion et les Antilles (“zouk affairs ”, “Intens" ”, “Karamell ”, “Émeraude”) ;

- le reggae aux consonances jamaïquaines très présentes (D'J' Dom, Aston Bernett, Peter Tosh) ;

- les groupes locaux comme entre autres Baster, Oussanoussava ;

- et, pour Maurice, le ségga et le séggaé (hybridation avec le reggae avec notamment le groupe Cassiya et les chanteurs Joël Vignes ou Kaya dont la disparition a déclenché les mouvements de 1998).

Ces genres musicaux peuvent s'entendre aussi bien à Maurice qu'à La Réunion : Cassya par exemple se donne en concert à St-

\footnotetext{
5 À Madagascar les maladies sont souvent encore interprétées comme l'intervention des ancêtres sanctionnant la transgression d'une norme sociale (Rajaonarimanana, 1999).
} 
Leu (La Réunion) pour la sortie de son dernier album " Racine de vie ", alors que Baster passe à Cure-pipe (Maurice) pour présenter le sien. Mais la musique et les vedettes malgaches (Mily Clément) sont moins présentes dans les hits-parade réunionnais et mauriciens. L'étude comparée des hitsparades dans les trois pays montre en retour que les chanteurs mauriciens et Réunionnais ne s'écoutent pratiquement pas à Antananarivo. Exception, faite du chanteur mauricien Kaya, mais qui est plus connu à Madagascar comme martyre que pour ses disques. Cependant il faut interpréter avec prudence les indications fournies par l'étude des hit parades qui reposent le plus souvent sur des palmarès de ventes. Il convient, en effet, de relativiser l'importance de la démarche d'achat en magasin qui ne constitue qu'un mode d'acquisition parmi d'autres des cassettes et des CD.

Il concerne surtout les jeunes les plus nantis, or sur les trois îles les autres disposent d'autres circuits d'approvisionnement (copies, échanges, marchés parallèles). Pourtant on peut avancer que l'écoute musicale à Madagascar privilégie deux dimensions : l'espace local avec des formes traditionnelles très vivaces (comme le Hira-gazy) et la dimension planétaire en faisant l'impasse sur la musique des autres îles. Les seuls dénominateurs communs musicaux qui restent alors au niveau de l'Océan Indien sont en définitive fournis par les idoles de la culture " Mc monde ". Madonna, Cher, Phil Collins, Ricky Martins et autres stars des variétés mondiales qui ont droit de cité dans l'ensemble des hit parades et souvent sur les murs des chambres des adolescent(e)s.
Cinquième erreur. Sur une même plage les différences foisonnent ; de même qu'il faut se garder d'amalgamer les jeunes de l'Océan Indien, il faut déjouer l'illusion faisant des jeunes de La Réunion, de Maurice ou de Madagascar une totalité univoque et unifiée. À ce point précis il est interdit de se tromper : chaque île repose sur la diversité, alors autant éviter de succomber à la tentation de construire des totalités culturelles. On ne peut pas écrire "les Réunionnais" comme certains ethnologues ont fait usage du "ils" ou du " eux" (collectif et globalisant) pour décrire "les " Nuers, "les ” Bororos, ou "les" Arapeshs. Tout tableau uniformisant gommerait la spécificité de ces îles qui tient en un paradoxe : le métissage culturel est d'ordinaire la conséquence de la mondialisation à laquelle on oppose la défense des identités locales, or ici le métissage est l'identité locale.

\section{Le sport : un exemple d'investissement} culturel à géométrie variable entre local et mondialisation

Rien ne semble devoir arrêter l'extension de la coopération régionale inter-îles, impulsée par la Commission de l'Océan Indien dans plusieurs domaines : transports, pêche, artisanat, échanges commerciaux ${ }^{6}$, coopération industrielle ${ }^{7}$, tourisme $e^{8}$, coopération scientifique et technique, santé9 , environne-

\footnotetext{
${ }^{6}$ Mise en place d'un cadre juridique visant l'abaissement des barrières tarifaires et l'aménagement des réglementations non tarifaires.

${ }^{7}$ Mise en place d'un Conseil régional de la coopération industrielle (CRIC).

${ }^{8}$ La direction régionale du tourisme (basée aux Seychelles) a pris la relève de l'Alliance Touristique de l'Océan Indien (ATOI) créée en 1966 et dissoute en 1977 faute de ressources.

${ }^{9}$ Mise en place d'un réseau régional de veille épidémiologique, plan d'action régional de secours en mer...
} 
ment, éducation. Ce dernier domaine constitue un remarquable secteur de concentration de la coopération ayant permis la mise en place d'une université de l'Océan Indien $\left(\mathrm{UOI}^{10}\right)$. Mais la vitalité des échanges de formations ne peut faire oublier les fragilités du bassin en terme d'échanges commerciaux ${ }^{11}$ intra-zone et de manque d'autonomie financière vis-à-vis du Fonds économique de développement (FED) et de l'Union européenne. Du coup, comme l'ont souligné Oraison et ses élèves (Oraison, 1988 ; Cadet, 1997) les visées premières d'une organisation internationale à vocation économique glissent alors vers des objectifs surtout symboliques de “bon voisinage "12. D'où une place importante accordée aux sports en général et aux jeux de l'Océan Indien en particulier pour développer et entretenir l'esprit de fraternité entre les différentes îles. Mais la cinquième édition de cette épreuve n'a pas semblé entraîner l'engouement des précédentes. On voit à cela plusieurs raisons.

- Premièrement, les Réunionnais, organisateurs en 1998, semblent avoir davantage les yeux tournés vers la métropole où s'exportent leurs meilleurs joueurs que vers les performances régionales. D'où en conséquence une dévalorisation relative des jeux des îles, et de leurs victoires jugées trop aisées. Les 30 victoires réunionnaises sur les 31 courses de natation sont régulièrement mises en avant dans les commentaires des jeux (ce qui masque d'ailleurs un bilan global plus équilibré13). D'une manière générale, les joueurs connus des autres îles sont ceux qui évoluent dans le championnat de football de La Réunion (par exemple les Malgaches) ou alors ceux qui évoluent dans les champion- nats de métropole (par exemple Hoffman le volleyeur mauricien). La presse sportive océanienne, destinée à mieux faire connaître les sportifs de la région, privilégie parfois la mise en exergue de ceux qui ont atteint la gloire en métropole et plus largement en Europe. Patrice Casimir (gymnastique), Laurent Robert (football), Jakson Ridcharson (handball) illustrent ce détour par lequel on devient vraiment une vedette sportive. La consécration passe par «l'expatriation ». L'universalisme dans le domaine du sport éclipse le régionalisme.

- Ensuite, l'engouement lors des premières éditions des jeux était à son comble parce que les nations et le département français en lice avaient encore un niveau de développement relativement proche, la compétition y figurait la passion pour l'égalité des chances. Au fil des éditions, l'essor économique de La Réunion a creusé un écart avec les autres îles. Du coup la compétition semble moins à armes égales quand La Réunion fait figure, vue des Maldives, des Comores ou des Seychelles, d'île surdéveloppée en infrastructures sportives et pouvant mobiliser une technologie de pointe pour l'entraînement de ses champions. Le sentiment d'injustice est avivé par la possible participation des sportifs

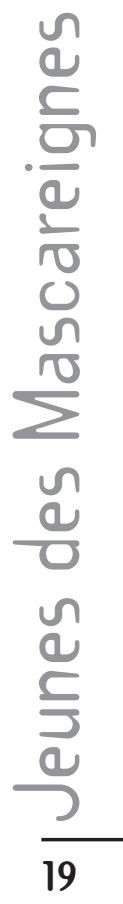

\footnotetext{
${ }^{10}$ Les grandes lignes en furent tracées en 1995 et elle a vu le jour en 1998, s'achève aujourd'hui le premier programme de formation sous la présidence de Goolam Mohamedbhai.

${ }^{11}$ Elle ne le cherche pas, mais bien à y pallier en formant des jeunes qualifiés.

12 Les objectifs " relèvent plus de relations de bon voisinage que d'une réelle coopération se traduisant par des échanges commerciaux substantiels" (Oraison, 1988).

13191 médailles pour La Réunion, 164 pour l'île Maurice, 137 pour Madagascar, 69 pour les Seychelles, 7 pour les Comores, 2 pour les Maldives. II est donc toujours un peu facile pour le supporter réunionnais d'oublier les contre-performances de ses champions en judo, tennis, volley quand Franck Schott ou Aurélie Gresset truste les médailles en natation.
} 


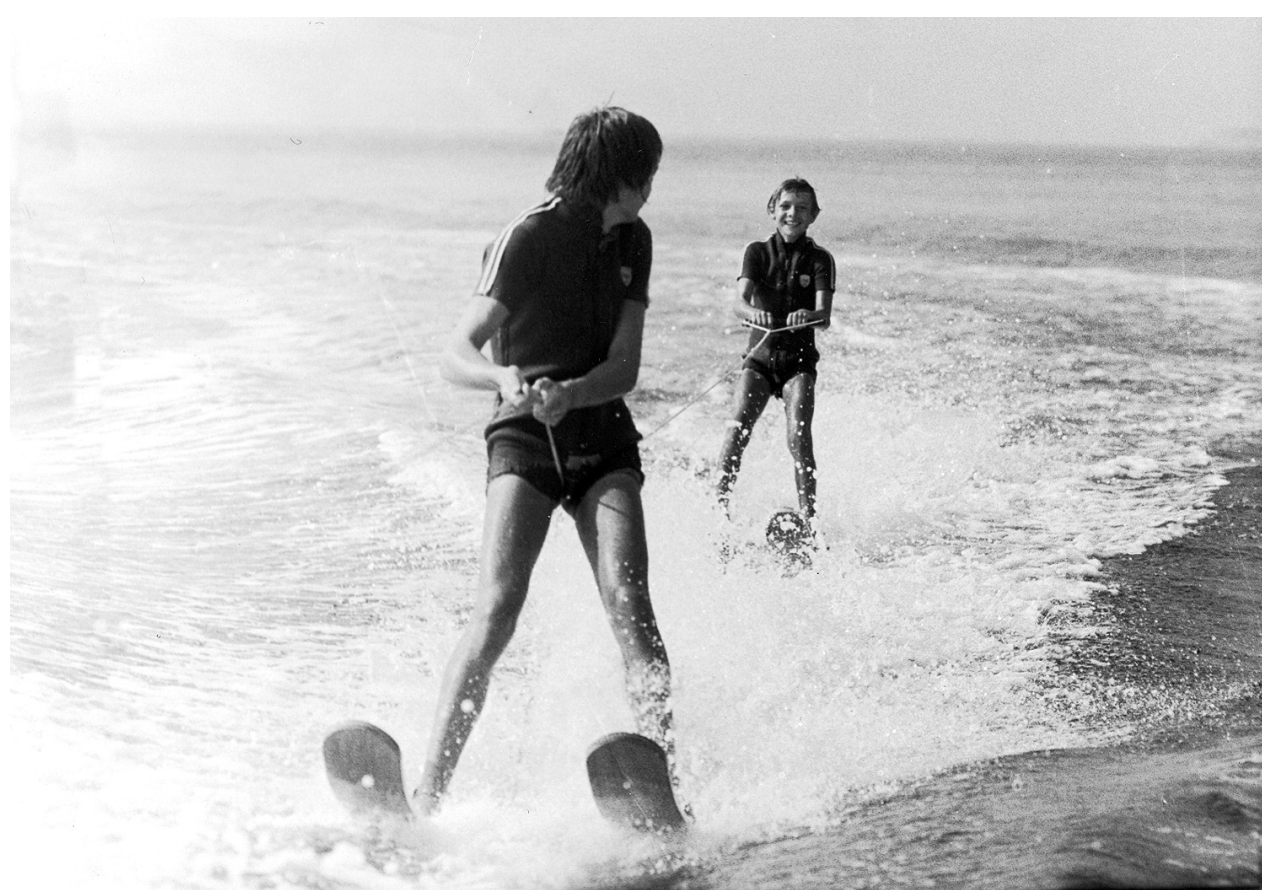

réunionnais à un championnat en métropole (d'où l'article 28 de la Charte des Jeux de l'Océan Indien limitant la participation des sportifs réunionnais n'évoluant pas sur l'île $\left.{ }^{14}\right)$.

- Enfin, les Jeux sont porteurs d'enjeux à géométrie variable. Pour les pays à l'économie la plus précaire comme les Maldives ou les Comores participer, c'est-à-dire manifester sa présence, revient à l'essentiel. Pour l'île de Ratsiraka, à cause de difficultés

\footnotetext{
${ }^{14}$ Article d'autant plus controversé par les supporters réunionnais qu'il permet aux îles qui sont aussi des États d'envoyer leur champions en préparation où elles l'entendent. Mauriciens et Malgaches peuvent évoluer en France sans avoir à renoncer aux jeux de l'Océan indien. L'exemple le plus polémique concerne l'interdiction de participation imposé à $F$. Giraudet volleyeur à Agde alors que son coéquipier Hoffman pouvait, lui, défendre les couleurs de Maurice.

15 Madagascar connaît d'importants foyers de lèpre et de tuberculose, l'espérance de vie y est d'une vingtaine d'année inférieure à celle de La Réunion.
}

économiques et d'une situation sanitaire encore problématique ${ }^{15}$, les jeux tiennent un rôle de vitrine de la bonne santé du pays. Enfin, pour La Réunion l'enjeu n'est plus uniquement dans le nombre de médailles remportées, l'épreuve se déplace dans le champ de l'organisation où il s'agit de faire preuve de son excellence et de son modernisme. D'où la référence appuyée et réitérée dans la presse à la création d'un site internet, à la sophistication des cartes d'accréditation ou enfin au modernisme du nouveau gymnase de St-André.

\section{Conclusion}

L'Océan Indien représente 75 millions de kilomètres carrés d'eau, desquels émergent quelques îles. Les jeunes qui y vivent connaissent le plus souvent des réalités cloisonnées. Le bassin culturel est donc plus à faire 
qu'une donnée de départ; il représente un beau pari à gagner (en favorisant la mobilité, les échanges...) pour offrir aux jeunes le privilège d'appartenir à plusieurs mondes (local, régional, mondial) dans une seule vie. Les obstacles ne manquent pas à sa réussite mais même Paris ne s'est pas fait en un jour.

\section{Bibliographie}

Barnabé, J., et al., Éloge de la créolité, Paris, Gallimard, 1989.

BRIAL, F., " La coopération internationale décentralisée des collectivités locales réunionnaises ", in Annuaire des pays de l'océan Indien 1995-1996, Aix, CNRS/P.U. d'Aix, 1998.

Cadet, F., La Commission de l'Océan Indien douze ans après 1984-1996, D.E.A. Université de La Réunion, 1997.

Chane-Kune, S., Aux origines de l'identité réunionnaise, Paris, L'Harmattan, 1993.

Chaudenson, R., Des hommes, des îles, des langues, Paris, L'Harmattan, 1992.

Chaudenson, R., Vers une révolution francophone? Paris, L'Harmattan, 1995.

Chaudenson, R., Les Créoles, Paris, PUF, 1995.

Cheynel, P., Images de la vieillesse : place et rôle de la personne âgée vue par des adolescents des Comores, Maurice, La Réunion, Seychelles, Madagascar et France métropolitaine, Université de La Réunion, 1985.

Commission de l'Océan Indien, Compte rendu des travaux du Comité régional des échanges commerciaux, 1994.

Gallani, C., Giochi pericolosi. Framenti di un immaginario razzista, Rome, Mlibri, 1996.

GHosh, R., “ L'influence de l'Inde dans l'océan Indien ”, Annuaires des pays de
l'Océan Indiens. 1992-1994, Aix, CNRS/P.U. d'Aix, 1995, p. 401-420.

Gerbeau, H., “Approche historique du fait créole à La Réunion ”, in Îles, insularité, insularisme, Bordeaux, C.R.E.T., Université de Bordeaux III, p. 125-156, 1987.

Gruzinski, S., La pensée métisse, Paris, Fayard, 1999.

Mathieu, J.-L., L'outre-mer français, Paris, P.U.F., Coll. Politique d'aujourd'hui, 1993.

Maestri, E., Les îles du Sud-Ouest de l'Océan Indien et la France de 1815 à nos jours, CDHR, Paris, L'Harmattan, 1994.

MiÈGE, J.-L., " Intendured Labour in the indian ocean and the particular case of Mauritius ", Intercontinenta, Leiden, $n^{\circ} 5$, p. 3., 1986.

ORAISON, A., Quelques réflexions critiques sur la commission de l'océan Indien : les obstacles à la coopération régionale dans la zone Sud-Ouest de l'Océan Indien, Université de La Réunion, 1988.

Rahamefy, A., " Crises religieuses et sectes à Madagascar ", Études de l'Océan Indien, $\mathrm{n}^{\circ} 21$, p. 7-105.

Rajaonarimanana, N., “ Les documents médicaux de la tradition du sud-est de madagascar ", Études de l'Océan Indien, n 19 , p. 11-44, 1995.

RAJAONAH, H., La coopération régionale entre les îles du Sud-Ouest de l'Océan Indien, DEA, Université de La Réunion, 1996.

Ramilison, S., Essai d'analyse du phénomène Dina Menavozo dans les régions du SudEst. Cas de Farafangana, École nationale d'administration malgache, Antananarivo, S.I.D., 1993.

Vellas, F., Cauet, J.-M., Le tourisme et les îles, Paris, L'Harmattan, 1997. 


\section{What are cultural identities for young people in the Indian Ocean?}

On the Etang Salé beach (Reunion), on Flic en Flac beach (Mauritius), on Praslin beaches (Seychelles) or even on the large coastal stretches close to Tamatave (Madagascar), the same young people seem to be playing the sames games while listening to the same music. Everything contributes to this lack of differentiation, aren't these island considered as « Sister islands? The aim of this paper is to invite the reader to take part in an unusual game of " spot the differences «, as an antidote against the standardized vision projected by cliches of deceptive exotism.

\section{¿Qué identidades culturales para los jóvenes del Océano Indico?}

En la playa de la Laguna Salada (Reunión), al igual que en la del «Flic en Flac» (Mauricio), como en las de Praslin (Seychelles), o también en las inmensas extensiones costeras cercanas a Tamatave (Madagascar), parece que veamos a los mismos jóvenes jugando a los mismos juegos y escuchando la misma música. Todo conspira a este efecto de falta de diferenciación, ¿no se habla de «islas hermanas»? El propósito de este artículo es, en primera instancia, invitar al lector a participar en un insólito «juego de

\footnotetext{
los cinco errores", como antídoto a la visión uniformizante proyectada por los tópicos de un exotismo engañoso.

22
}

\section{Welche kulturelle Identitäten Für Jugendliche aus dem Indischen Ozean?}

Am Strand von Etang Salé (Réunion) wie am Strand von Flic en Flac (Mauritius) oder am Strand von Praslin (Seychellen) sowie an den riesengrossen Küstenstreifen in der Nähe von Tamatave (Madagascar) sind es anscheinend die gleichen Jugendlichen, die die gleichen Spiele haben und dabei die gleiche Musik hören. Alles trägt zu dieser Undifferenzierungswirkung bei, spricht man nicht übrigens von den «Schwesterinseln «? Ziel dieses Artikels ist zuerst den Leser an einem «Spiel der fünf Irrtümer « teilnehmen zu lassen und zwar als Gegenmittel zur vereinheitlichenden Vision, die von den Klischees einer teuchenden Exotik hervorgerufen wird. 